\title{
Dynamic Displays: the Changing Face of Augmentative Communication
}

\author{
Walter S. Woltosz \\ Wordst, Inc. \\ Palmdale, California, 93550 USA
}

\begin{abstract}
Augmentative and altemative communication (AAC) devices incorporating dynamic displays have been commercially-available since 1981 for text-based systems, and since 1986 for graphic-based systems. Beginning in about 1990, the advantages of dynamic displays became so overwhelming and so obvious that clinicians began recommending them more frequenty and their use increased rapidly. This paper reviews the history of dynamic display AAC devices, describes their advantages and disadvantages with respect to static display systems, and discusses relevant reseach literature.
\end{abstract}

\section{Introduction}

"The purpose of a communication display is to arrange language in space so individuals can, by selecting from the available options, say what they wish to say as quickly as possible, and can do so with a minimal amount of effort." [1]

The above quotation from Sarah Blackstone is profound. For AAC users with sufficient visual skills to use visual feedback, the display of language symbols is often the primary factor that determines both the speed of communication and the amount of language available. From simple picture boards, to complex symbol boards, to letter/word boards, to electronic devices with fixed (static) symbols, to electronic devices with dynamic displays, those who have had the task of selecting the symbols to be displayed and their arrangement have struggled to provide access to large amounts of language with displays that are easy to use.

\section{The Early Years - Static Displays}

The earliest AAC devices were communication boards and books, and these remain in widespread use. Symbols used on such boards and books include small objects, tactile surfaces (sandpaper, cotton, cloth, etc.), photographs, pictographic symbols (line drawings, either in black and white or in color), abstract symbols, letters, words, and phrases. If the user can turn pages, then a communication book is often used to allow access to more than one page of symbols. If not, a single communication board is used with a reduced symbol set. For users with visual impaiments and/or with limited pointing accuracy, symbols must be made large, further reducing the number of symbols that can appear on the display. Thus, language often has to be compromised in favor of visibility or accessibility. 


\subsection{Limited Symbol Sets}

The greatest language limitation in early devices stemmed from the limited symbol set. This was especially true for users of pictographic symbols, but was also experienced by text users who used not only the alphabet, but complete words and phrases on their boards to save time.

Pictographic Symbols. Displays made up of pictographic symbols are used for nonspeaking persons who are able to see, but who are unable to spell or read. With these "low tech" devices, the user simply indicates (by pointing, or by some kind of signal when someone else does the pointing) a choice of one or more picture symbols in order to communicate a message. The symbols are selected to be easily recognized, and each is associated with a single language concept. With this method, the user relies on recognition memory - a relatively low cognitive function - in order to associate the picture with the language it represents [2].

Clinicians and teachers who make picture boards and picture books find them relatively easy to construct, inexpensive, and effective (at least for the symbols that are on the display. Note that the term "picture" is used here to mean pictographic symbols, as well as actual photographs.).

Adding spcech output. During the early 1980's, electronic systems with synthesized speech output were developed for pictographic communication. Shortly thereafter, systems with recorded speech/sounds began to appear. These systems used keyboards and/or LED displays with picture symbols on or next to each key or LED. Most systems allowed no more than 128 pictures, although many users of such systems had much larger vocabulary and language.

Just as with earlier paper communication boards (but less so with communication books), there was a practical limit to the number of symbols a user could have (or see, or reach) within the size of the display area. The picture book got around this limitation to some extent by allowing the user to have many pages of symbols, allowing the same space to be occupied by various sets of symbols by turning pages; however, turning pages was not always practical, and the number of pages was usually limited by size, weight, and ease of finding the desired page when large numbers of pages were used.

Static display strategies. Static displays are those on which the language symbols do not change as the user operates the device. A static display is usually a paper or plastic sheet containing a number of language symbols arranged in some way typically in rows and columns, but sometimes in a circulat pattern, a single line, or some other arrangenent.

The problem with static displays arises when the user's vocabulary (i.e., the number of language items in the user's repertoire) exceeds the number of locations for symbols 
on the display. In order to represent all of the user's language with the limited symbol set, some form of multiple use of symbols - an encoding scheme - is required.

Two such strategies have been employed: (1) levels, and (2) symbol sequences. Nearly every manufacturer of AAC devices has used both strategies. Some manufacturers have assigned special names to their particular implementation of these strategies for marketing purposes. Each of these strategies enables more than one action to be represented per symbol - millions of language elements can theoretically be represented with just 128 symbols. Both strategies are also often used together.

Levels. Using the concept of levels, the display appears as a single set of symbols, but there are actually two or more "levels" to the display, somewhat as though there were two or more devices, each with its own set of messages. Thus, a symbol has different meanings on different levels. In operation, the user first selects the desired level, and then selects the symbol(s) needed to communicate the message. As an example, a DRINK symbol might represent "I'd like a drink of water" on Level 1, "I'd like some milk" on Level 2, "May I please have some orange juice" on Level 3, and so on.

The primary difficulty with this approach is that the user had to employ recall memory to remember which level contained the desired message, then again use recall memory to remember how to get to the desired level, then select the level, and then select the symbol(s). Recall is a higher cognitive function than recognition [2], so the level strategy increased the cognitive load on the user. In fact, many devices capable of providing multiple levels are often actually used with only a single level - users are not always able to use the full capacity of the device because the cognitive load is too high.

In addition to the cognitive load, there is a high visual perceptual load with static displays resulting from the fact that all of the user's symbols (up to 128) are always in view. In this instance, the visual perceptual load refers to the quantity of visual information presented to the user. In a field of many symbols, the amount of information is high, and the user must search the dense visual field to find the desired symbol. Visual perceptual load is increased when the number of symbols is high, when symbols are very similar in appearance, when symbols are closely packed together, when symbols have multiple semantic meanings, and when symbols contain much fine detail and/or background information. For users with limited visual perceptual skills, the number of symbols may have to be reduced, and the size and/or spacing of the symbols may have to be increased, further limiting the number of symbols available to represent the user's language on a static display.

Sequences. The second strategy that is used to let a limited set of symbols represent a larger set of language items is symbol sequencing. This strategy was used thousands of years ago by the Chinese, Egyptians, and Mayans, among others. Symbol sequencing takes advantage of the many ordered combinations of symbols possible with a limited symbol set. For example, a set of only eight symbols can produce 64 
possible ordered pairs of symbols. With 26 symbols (the number of letters in the alphabet) a total of 676 ordcred pairs are available, while 128 symbols can produce 16,384 ordered pairs. With 128 symbols and allowing up to three symbols in sequence, over two million combinations are possible.

With pictographic symbol sequencing, the user might select a SUN symbol followed by a symbol for DRINK to represent their favorite hot drink - e.g., select SUN, then select DRINK, and the device would say "I'd like some hot chocolate". Or the user might select a TREE symbol followed by the DRINK symbol to say "I'd like some apple juice" (if there was not an APPLE symbol but there was a tree symbol).

These sequences probably seem logical to you, because you have the world knowledge to know that the sun is hot and that apples grow on trees (of course, so do lemons, oranges, pears, grapefruit, various kinds of nuts, Spanish moss, mistletoe, and lots of other things!). But for many users of pictographic symbols, world knowledge is still in an early developing stage. You need to have world knowledge to use the language system, but you need a language system to acquire world knowledge. Thus, this attempt to make it easier to recall symbol sequences actually can result in the requirement for rote memorization of what seem to be abstract sequences to the user.

Some developers have put a great deal of effort into coming up with associations that are supposed to be easy to remember, but which in practice have proved quite difficult and even less effective than simple letter abbreviations (for those who are capable of using letter abbreviations [3]). A picture of an elephant might carry the connotations of "big", "gray", "memory" (because "elephants don't forget"), "animal", and other meanings, depending upon the context in which the symbol was used with other symbols. A SUN symbol might mean "hot", "big", "round", "yellow", "far away", "morning", "day" and more. The tremendous amount of world knowledge required to make such associations, as well as the varying associational rules (size, color, shape, distance, temperature, time, cognitive characteristics, etc.), impose perhaps the most demanding cognitive load ever developed for augmentative communication system users, requiring hundreds of hours of training to achieve a reasonable level of communicative competence.

In the past, these demands on the user were necessary because of the limited symbol set - a restriction inherent in the available techologies associated with static displays. The computing power of early devices simply could not support anything more sophisticated, and so the user had to do more of the work. With enough computing power, as is now readily available, there is no longer any reason to impose these high loads on the user.

\section{Dynamic displays - the future is now}

A dynamic display is one which can present a changing set of symbols to the user. 
Computer screens are dynamic displays, whether on a desktop CRT or on a notebook computer with an LCD (liquid crystal display).

The advent of dynamic displays has complstely changed the preferred approach to augmentative communication for most AAC users. Modern systems allow rapid changing of the displayed symbolic elements in accordance with simple language rules. This makes possible a large symbol set, eliminating the need for multi-meaning symbols and allowing direct association between a symbol and the language or action it represents. At the same time, while the total number of symbols becomes unlimited (for all practical purposes on the most powerful dynamic display systems), the number of symbols displayed at any one time can be kept to a minimum in order to reduce the visual perceptual load. The user might have many thousands of symbols in their system, but might see only a dozen or two at any one time. The reduced cognitive load, reduced visual perceptual load, and reduced visual acuity load all mean faster and easier learning and faster and easier selection, which translate into faster and easier communication.

Large dynamic displays allow the system to behave like an electronic "communication book", much like the plastic-paged books often used for beginning picture communicators. For picture users of dynamic displays, for example, the software driving the display allows the user to select any of a large number of "pages", such as a food page, a drinks page, a places-to-go page, a pcople page, and so on. The large number of pictures allows more of a one-to-one association between the symbol and the language it represents - eliminating the need to memorize levels, as well as the need for multi-meaning symbols or abstract symbol sequencing.

Consider the DRINK symbol mentioned earlier in the discussion on levels. Rather than remembering that if Level 1 is selected and then the DRINK symbol is selected, the device will say "May I have a drink of water", and if Level 2 is first selected and then the DRINK symbol is selected, the device will say "I'd like some milk", and so on, when you use a dynamic display device you might simply choose the DRINK symbol and the screen would change to a page of drinks - with explicit symbols for each of the drinks you might want to select. If you want milk, you select the milk symbol. If you want orange juice, you select the orange juice symbol, and so on. The association is direct, and the process is self-prompting, relying on recognition memory, and eliminating the need for rote memorization and recall memory.

\subsection{Symbols and the language they represent}

With both static and dynamic displays, the choice of symbols for a particular AAC user, and their arrangement, is crucial. The "canned language" approach of providing a set of symbols and the language that goes with them, and then trying to teach this "language" to the user, is just the opposite of what published research indicates should be the preferred approach - to base the selection of symbols, and how you organize them, on the user's associational and categorizational strengths [4]. 
In a recent study of the vocabulary of preschool children, not one single word was found to be common to the vocabulary of the 90 test subjects (the word "Mom" was in 85 of the 90 , and the top $10 \%$ of the items were only common to 18 of the 90 subjects) [5]. This illustrates the need to tailor the vocabulary (symbols) to the needs of the user, rather than using someone's preconceived idea of what the user's language should be. There are no standard vocabularies for any population of AAC users.

Cognitive science, a field only a few decades old, is investigating many aspects of how we think, including how we organize information in our brains [6]. People organize language in different ways, including schemalic, taxonomic, semanticsyntactic, and alphabetic [1], and the specific organization that works best for one individual may be radically different from that for someone else. Thus, there are no standard categories.

If there are no standard categories, and there are no standard vocabularies, then what is a clinician to do to prepare a communication system for a user? The answer goes back to the earlier reference regarding the user's associational and categorizational strengths. Clinicians simply must use their training and skills to determine the user's language, and then program that language into the $A A C$ device. We need to be putting users' language into devices, not devices' language into users.

\subsection{Transition to literacy}

One of the major issues in the field of augmentative communication today is the transition to literacy of users of pictographic communication systems. More abstract pictographic communication systems have been criticized for the detrimental effects they have been observed to have on the language development of their users, particularly with respect to learning literacy skills.

The dynamic display, properly implemented, can provide a system capable of using pictographic symbols and traditional orthography at the same time - mixing pictures and words in any proportion and any order. In fact, the technology of changing pages of symbols to which speech output can be assigned provides new opportunities for developing powerful tools to facilitate learning a variety of concepts, including literacy skills, mathematics, and more.

\subsection{Dynamic displays and text-based communication}

Dynamic displays are most often thought of in connection with pictographic communication; however, they were first used for text-based communication, and their advantages for text-based users have grown tremendously since their introduction in 1981. As with pictographic language, text-based language benefits from reduced cognitive and visual perceptual loads, expanded vocabulary, ease of learning, and ease of use. 
The earliest use of the dynamic display for text was to offer word and phrase lists from which the user could select in order to minimize spelling. In the Words+ Living Center of 1981, for example, the user could select any of a set of high frequency words directly from the primary screen display, and could then further select any of about one thousand additional words by first selecting the first letter of the desired word, and then selecting from pages of words beginning with that letter. This early system, based on a $64 \mathrm{~K}$ computer, provided single switch communication at rates up to ten words per minute for proficient users. Beginning in about 1990, with memory and disk storage measured in megabytes and hundreds of megabytes, text-based users have been able to access many thousands of words and many hundreds of thousands or millions of phrases with a few selections - and with very little need for recall memory beyond basic spelling skills. Fron second graders to Professor Stephen Hawking, text-based users are communicating more quickly and easily than ever with dynamic displays.

\subsection{Technologies required for dynamic displays.}

Effective use of a dynamic display requires much greater computing resources than for static displays. These resources are: display size, memory (chips), mass storage (e.g., disk drives), and speed. It is not enough to have a large display - you must also have the computing "horsepower" to drive it properly. Some early devices had only $48 \mathrm{~K}$ of memory $(49,152$ bytes). On modern color displays, the memory required to represent a single screen of pictographic symbols can be over 160,000 bytes. And other resources are similarly taxed.

For example, the color Talking Screen pictographic communication software from Wordst, requires approxinately 15 million bytes of disk storage, at least four megabytes (over 4,000,000 bytes) of memory, and sufficient computing speed to allow drawing an entice screen of color pictures in a fraction of a second. Some "modern" dedicated devices with similar price tags have only a fraction of a percent of the computing capacity of such a computer-based dynamic display system.

The advances in technology are not just window dressing - they make dramatic differences in the kinds of augmentative communication strategies available to users, as well as for those who program the systems for the users. If the computer is not fast enough, then the user would have to wait too long before a new page of pictures would be drawn before making another selection. If there is not enough memory or mass storage, then the number of picture symbols available to the user is reduced, and the ease of programming the system is compromised. If the display size is not big enough, then the number and/or size of pictures the user can have on a page is limited. Color, which is now available on dynamic display devices, provides additional information making pictographic symbols more transparent. (easily recognized), and which has been shown to be necessary for some populations to recognize some symbols [7]. 


\section{Summary}

Dynamic display AAC systems incorporate state-of-the-art technologies to reduce cognitive and visual perceptual loads, and to decouple the language aspects of the device from the motor and visual requirements, while simultaneously increasing the user's access to language and decreasing the learning costs of using the system.

All of these technologies are important in directly affecting the augmentative communication strategies available to the user (which results in making the machine do as much of the work as possible).

For both pictographic and text-based AAC, dynamic displays are the future, and the future is now!

\section{References}

1. Blackstone, S. (1993) Thinking a little harder about communication displays. Augmentative Communication News 6:1.

2. Light, J., \& Lindsay, P. (1991) Cognitive Science and Augmentative and Alternative Communication. Augmentative and Alternative Communication (AAC), $7(3), 186-203$.

3. Light, J., Lindsay, P., Siegel, L., \& Parnes, P. (1990) The effects of three message encoding techniques on recall by literate adults using AAC systems. Augmentative and Alternative Communication (AAC), 6(3), 184-202.

4. Beukelman, D., Yorkston, K., \& Dowden, P. (1985) Communication Augmentation: A casebook of clinical management. San Dicgo, CA: College Hill.

5. Fried-Oken, M., \& More, L. (1992) An initial vocabulary for nonspeaking preschool children based on developmental and environmental language sources. Augmentative and Alternative Communication (AAC), 8(1), 41-56.

6. Lakoff, G. (1987) Women, Fire, and Dangerous Things, Chicago, IL. University of Chicago Press.

7. Mineo, B. (in preparation) Pictorial representation: An analysis of the abilities of two young adults with severe mental retardation. 